\title{
RISK FACTORS OF CHILD OBESITY IN UKRAINE
}

\author{
M. Aryayev, I. Tsyunchyk, S. Dhakre \\ Pediatrics and Neonatology, Odessa National Medical University, Odessa, Ukraine
}

Background and aims: To detect the risk factors of child obesity and to give recommendation for prevention of obesity in population.

Methods: Patients admitted to endocrinological department who met the inclusion criteria were interviewed. The inclusion criteria were: weight excess / obesity exogenous-constitutional or hypothalamic, age $\geq 3$ year, ability to give informed consent. Exclusion criteria were: Cushing disease / syndrome, Turner syndrome.

Using a standardized protocol, families and children were interviewed according to the corresponding questionnaire. Data entry and descriptive analysis were undertaken with Epi Info. To evaluate the relative risk, the univariate and multivariate logistic regression analysis were used and its interpretation was expressed in odds ratio's (OR) with confidence interval (CI) of $95 \%$.

Results: Risk factors associated with compromised inheritance of children and families include: Obesity / Methabolic syndrome $X(\mathrm{OR}=16,01 ; \mathrm{CI}=7,20-35,61)$, Hypertension $(\mathrm{OR}=1,86 ; \mathrm{CI}=0,94-3,67)$, Diabetes mellitus 2 type $(\mathrm{OR}=3,77 ; \mathrm{CI}=1,70-8,35)$, Pathology of reproductive system $(\mathrm{OR}=2,28 ; \mathrm{CI}=1,11-4,66)$. Among the risk factors associated with perinatal period there are: severe gestosis during pregnancy $(\mathrm{OR}=3,00 ; \mathrm{CI}=0,86-10,45)$, powerless labor $(\mathrm{OR}=3,13$; $\mathrm{CI}=1,45-6,75)$, low birth weight (less than $2800 \mathrm{~g})$ $(\mathrm{OR}=5,70 ; \mathrm{CI}=2,78-11,69)$, artificial feeding $(\mathrm{OR}=5,10 ; \mathrm{CI}=2,14-12,11)$. The risk factors associated with the personality of a child include the peculiarities of the child's behavior.

Conclusions: The practical recommendations directed on elimination of the risk factors for the obesity prevention. 